\title{
EXTRACTION OF THE GENERAL SOLUTION OF INTEGRO-DIFFERENTIAL EQUATION IN GAUSSIAN ELECTRON BEAM
}

\author{
Haider M. Al-Juboori ${ }^{1}$, Mohammed Z. Al-Faiz ${ }^{2}$ and Raad A. Khamis ${ }^{3}$ \\ 1 College of Computer Sciences \& Math, Tikrit University, Tikrit, Iraq \\ 2 Computer Engineering Department, Al-Nahrain University, Baghdad, Iraq \\ 3 Applied Sciences Department, University of Technology, Baghdad, Iraq
}

\begin{abstract}
The fundamentals of free electron laser (FEL) theory are now well-established and can provide a sophisticated description of experiments over a wide range of parameters. While new technology is being developed for systems working from $1 \mathrm{~mm}$ to $10 \mathrm{~nm}$ wavelengths, the theory remains the same. In this work, the final consequences of Gaussian electron beam in FEL amplifier will be found by solving of Integro-differential equation using symbolic toolbox services in MATLAB. and the relation between several parameters will be delimit and plot in order to extracted and presented the complex relations between the FEL parameters.
\end{abstract}

Keywords: Evolutionary programming and Symbolic Analysis, Mathematical Modeling, FEL Amplifier Process

\section{INTRODUCTION}

The problem of electromagnetic wave amplification in the undulator refers to a class of self-consistent problems. It can be separated into two parts: Solution of the dynamical problem and Solution of the electro-dynamic problem.

To enclose the problem, the field equations and equations of motion should be solved simultaneously. In principle, modern HighPerformance computers allow one to perform direct simulation of the FEL process. The results of such simulations depend on a large number of problem parameters. They provide the possibility of obtaining a numerical answer for a specific set of input data.

A deeper insight into FEL physics can be obtained only by introducing some simplifying assumptions about the of the lectron beam and of the electromagnetic field.
Theoretical investigation of the free electron laser should be performed in two stages. In the beginning one should study the general properties of the FEL, namely the ideal mechanism of amplification. At the next stage different complications can be introduced into the FEL model allowing it to extend the number of additional effects influencing the operation of the FEL amplifier.

These factors can be divided into two groups; the space charge effects and diffraction effects are fundamental. On the other hand, there are a lot of other factors such as energy spread effects, non-ideality of the undulator field, etc. The principal difference of the fundamental effects and all the others is that the fundamental effects depend on the same physical parameters as the ideal FEL amplification mechanism itself. 
The one-dimensional model is an important one from the methodological point of view. The following assumptions are used in the one-dimensional, steady-state model of the FEL amplifier for linear region:

- The electron beam has Gaussian density distribution in the direction perpendicular to the undulator axis;

- The electron moves along identical trajectories parallel to the undulator axis;

- The amplified wave is a monochromatic plane wave; and

- The electron beam is infinitely long.

These suppositions will be the basic step in our mathematical treatments in the following work.

\section{THEORY}

The description of the Free Electron Laser (FEL) given here is based on Kim [1], Reiche [2] and Zangrando [3]. A more detailed description can be found in [4] and [5] and the references therein.

Beside the previous assumption, the treatment in this paper will be considered a monoenergetic electron beam (cold electron beam) as:

$$
\tilde{f}_{o}(P)=n_{e} \delta(P)
$$

where $P=\varepsilon-\varepsilon_{o}$, is the energy deviation.

The final format of integro-differential equation can be presented in following equation for the sequence of the integration can be interchanged, which describes the radiation field amplitude $\tilde{E}_{x}$ produced by an electron bunch with initial energy distribution $\tilde{f}_{o}(P)$ of $[4]$ :

$$
\begin{gathered}
\frac{\partial \tilde{E}_{x}(z)}{\partial z}=-\frac{\mu_{o} c^{2} \hat{K} e}{4 \gamma} \int_{0}^{z} \int_{-\infty}^{\infty}\left[\frac{e \hat{K}}{2 m_{e} c^{2} \gamma^{2}} \tilde{E}_{x}\left(z^{\prime}\right)\right. \\
\left.-\frac{e}{m_{e} c^{2}} \frac{2 c}{\omega \hat{K}} \frac{\partial}{\partial z^{\prime}} \tilde{E}_{z}\left(\mathrm{z}^{\prime}\right)\right] n_{e} \\
\frac{\mathrm{d} \delta(P)}{\mathrm{d} P} e^{-2 i k_{w} P\left(z^{\prime}-z\right)} \mathrm{d} P d z^{\prime}
\end{gathered}
$$

And the derivative of the ${ }^{\mathrm{TM}}$-function can be replaced by partial integration:

$$
\begin{aligned}
\int_{-\infty}^{\infty} F(P) \frac{\mathrm{d} \delta(P)}{\mathrm{d} \eta} \mathrm{d} P & =|F(P) \delta(P)|_{-\infty}^{+\infty} \\
& -\int_{-\infty}^{\infty} \frac{F(P)}{\mathrm{d} P} \delta(P) \mathrm{d} P \\
= & -\left.\frac{d F(P)}{d P}\right|_{P=0}
\end{aligned}
$$

Thus

$$
\begin{aligned}
& \frac{\partial \tilde{E}_{x}(z)}{\partial z}=- \frac{\mu_{o} c^{2} \hat{K} e}{4 \gamma} \int_{0}^{z} 2 k_{w}\left(z^{\prime}-z\right) \\
& e^{-2 i k_{w} P\left(z^{\prime}-z\right)}\left[\frac{e \hat{K}}{2 m_{e} c^{2} \gamma^{2}} \tilde{E}_{x}\left(z^{\prime}\right)\right. \\
&\left.+\frac{e}{m_{e} c^{2}} \frac{2 c}{\omega \hat{K}} \frac{\partial}{\partial z^{\prime}} \tilde{E}_{z}\left(\mathrm{z}^{\prime}\right)\right] d \mathrm{z}^{\prime}
\end{aligned}
$$

Introducing the gain parameter $\Gamma$ :

$\Gamma=\sqrt[3]{\frac{\mu_{\mathrm{o}} \hat{K}^{2} e^{2} k_{w} n_{e}}{2 m_{e} \gamma^{3}}}$

and a wave number (which can be identified with the wave number of longitudinal plasma oscillations):

$\Lambda_{p}=\sqrt{\Gamma^{3} \frac{4 c \gamma^{2}}{\omega \hat{K}^{2}}}$

equation (4) may be rewritten as:

$$
\begin{aligned}
& \frac{\partial}{\partial z} \tilde{E}_{x}(z)=-\Gamma^{3} \int_{0}^{z} i\left[\tilde{E}_{x}\left(z^{\prime}\right)+\frac{k_{p}^{2}}{\Gamma^{3}} .\right. \\
& \left.\frac{\partial}{\partial z} \tilde{E}_{x}\left(z^{\prime}\right)\right]\left(z^{\prime}-z\right) \mathrm{d} z^{\prime}
\end{aligned}
$$


This integro-differential equation can be transformed to a third-order differential equation [5]:

$$
\begin{array}{r}
\frac{\mathrm{d}^{3} \tilde{E}_{x}(z)}{\mathrm{d} z^{3}}-4 i k_{w} \frac{\mathrm{d}^{2} \tilde{E}_{x}(z)}{\mathrm{d} z^{2}}+\left(\Lambda_{p}^{2}-4 k_{w}^{2}\right) \\
\frac{\mathrm{d} \tilde{E}_{x}(z)}{\mathrm{d} z}-i \Gamma^{3} \tilde{E}_{x}(z)=0
\end{array}
$$

The above equation will be the fundamental point to numerical investigation in the next step.

\section{RESULTS AND DISCUSSION}

The differential equation, which is described in Eq.(8), can be solved by incorporate symbolic computation into the numeric environment of MATLAB for universal case or at $\left.\right|_{o}=0$ and small space charge forces $\left(n_{e}\right.$ small $\left.\Rightarrow \Lambda_{p}^{2} \approx 0\right)[6,7]$ :

$$
\begin{array}{r}
\tilde{E}_{x}(z)=A_{1} \exp (-i \Gamma z)+A_{2} \exp \left(\frac{i+\sqrt{3}}{2} \Gamma z\right) \\
+A_{3} \exp \left(\frac{i-\sqrt{3}}{2} \Gamma z\right)
\end{array}
$$

For $\mathrm{z} \rightarrow \infty$, the second term dominates. Th is indicates the onset of a collective instability in the internal charge distribution in the bunch. The electric field grows exponentially as $\exp (\sqrt{3} / 2 \Gamma z)$, the power as $\exp (\sqrt{3} \Gamma z)$.

For the realization, it can be found the general solution of Eq.(2) for a mono-energetic beam using the Laplace transform technique $[4,8,9]$ and MATLAB symbolic toolbox [10]. The same result can be extract using another technique, which the resultant cubic equation (in normalized form) can be written as [2]:

$\left((\lambda+\hat{C})^{2}-\hat{\Lambda}_{p}^{2}\right) \lambda+1=0$

Because the parameters $\hat{C}$ and $\hat{\Lambda}_{p}^{2}$ are real the cubic equation has always three solutions being either three real numbers or one real number and a pair of complex conjugate numbers. Each solution of $\lambda$ defines a mode for the evolution of the radiation field in time as $\widetilde{E}(z) \propto \exp (i \lambda \hat{z})$. The general solution is the sum of all three independent solutions which are found as following:

$$
\begin{aligned}
\lambda_{1}= & \frac{1}{6} \sqrt[3]{\boldsymbol{\alpha}}-6 \boldsymbol{\beta}-\mathrm{i} \frac{2}{3} \hat{\mathrm{C}} \\
\lambda_{2,3}= & -\frac{1}{12} \sqrt[3]{\boldsymbol{\alpha}}+3 \boldsymbol{\beta}-\frac{2}{3} \hat{\mathrm{C}} \\
\mp \mathrm{i} & \frac{\sqrt{3}}{2}\left(\frac{1}{6} \sqrt[3]{\boldsymbol{\alpha}}+6 \boldsymbol{\beta}\right)
\end{aligned}
$$

where;

$$
\begin{aligned}
\boldsymbol{\alpha}= & -72 \hat{\mathrm{C}} \hat{\Lambda}_{p}^{2}+8 \hat{\mathrm{C}}^{3}-108 \\
& +12 .\left(-12 \hat{\Lambda}_{p}^{6}+24 \hat{\Lambda}_{p}^{4} \hat{\mathrm{C}}^{2}-12 \hat{\Lambda}_{p}^{2} \hat{\mathrm{C}}^{4}\right. \\
& \left.+108 \hat{\Lambda}_{p}^{2} \hat{\mathrm{C}}-12 \hat{\mathrm{C}}^{3}+81\right)^{1 / 2} \\
\boldsymbol{\beta}= & \frac{-3 \hat{\Lambda}_{p}^{2}-\hat{\mathrm{C}}^{2}}{9 \sqrt[3]{\boldsymbol{\alpha}}}
\end{aligned}
$$

In the case of the solution with three real values for $\lambda$ each independent mode changes only its phase but not the amplitude. However the observed power $P \propto|\tilde{E}(\hat{z})|^{2}$ is fluctuating due to the interference of the three modes. The low gain FEL exploits this feature, and despite the interference, the absolute power of the radiation field and therefore the maximum gain of the FEL is limited to the order of the initial seeding field. Of particular interest is a solution of one real and two complex values for $\lambda$. The mode belonging to the real solution is oscillating while the others are exponentially decaying and growing. This is the phenomenon of a collective instability. At the beginning of the undulator all modes have almost identical amplitude and the driving mechanism is the interference of these mode, defining the 'start-up' regime.

After a certain distance the growing mode dominates showing an exponential amplification of the initial field. This is the 
typical characteristic of a high gain FEL, where the 'start-up' regime is succeeded by the 'exponential' regime. To avoid confusion the term 'exponential' refers only to the growth of the radiation field. Start-up and exponential growth are still given by the same differential equations of the FEL-model. Because this model used only the linear terms, both regimes of the radiation field evolution are combined to the 'linear' regime of the FEL.

If the normalized radiation field $\tilde{E}$ has grown to an amplitude of approximately 0.1 the non-linear terms in the differential equations are not negligible anymore [3]. Numerical simulation are showing that the radiation field will be limited to amplitudes of the order of unity. The exponential growth ends in this so called 'saturation' regime.

The growth rate $\mathfrak{R} e(i \boldsymbol{\lambda})$ in the linear regime depends only on two independent parameters: the detuning $\hat{C}$ and the space charge parameter $\hat{\Lambda}_{p}^{2}$.

The dependence of $\operatorname{Re} \hat{\Lambda}$ on $\hat{C}$ is shown in Figure (1). This result is quit similar to those presented in FEL literature [2,4]. For calculation purpose the space charge parameter has been set to zero, one, two, and three. The region of exponential amplification exists up to $\hat{C}<\hat{C}_{T h} \approx 1.89$ at $\hat{\Lambda}_{p}^{2}=0$ (where $\hat{C}_{T h}$ is the threshold detuning).

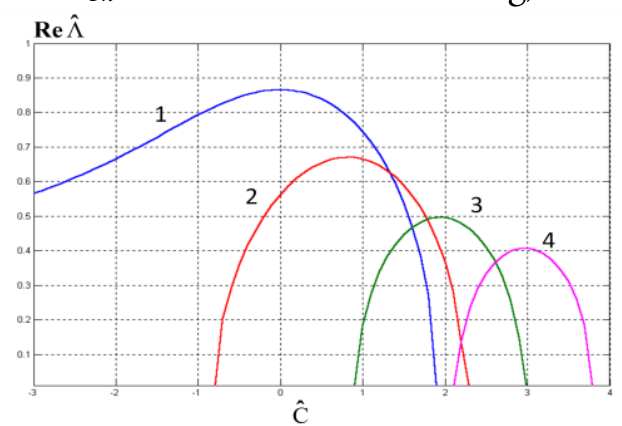

Fig.1. Reduced field growth rate $\mathbf{R e} \hat{\Lambda}$ versus the detuning parameter

$\hat{\mathrm{C}}$. Curve 1: $\hat{\Lambda}_{p}^{2} \rightarrow 0$. Curve 2: $\hat{\Lambda}_{p}^{2} \rightarrow 1$. Curve 3: $\hat{\Lambda}_{p}^{2} \rightarrow 4$. Curve 4: $\hat{\Lambda}_{p}^{2} \rightarrow 9$.
In the limit of a low current, the cubic dispersion relation (eq.10) is reduced to $(\lambda+\hat{C})^{2} \lambda+1=0$. The maximum growth rate occurs for $\hat{C}=0$ with $\mathfrak{R} e(i \lambda)=\sqrt{3} / 2$. This result seems to be in contradiction with the result of the low gain FEL, where at resonance energy no gain is visible. The explanation of this difference is that the low gain FreeElectron Laser remains in the start-up regime with $\hat{z}<<1[2]$.

The general solution $\tilde{E}$ for an unbunched electron beam and an initial seeding field $E_{\text {ext }}$ is

$$
\tilde{E}(\hat{z})=E_{e x t} \sum_{j} \frac{\exp \left(\lambda_{j} \hat{z}\right)}{1-2 i\left(\lambda_{j}+i \hat{C}\right) \lambda_{j}^{2}}
$$

where:

$\lambda_{j}$ are the roots of equation (11).

Now, the field gain can be presented as $E / E_{e x t}$, where $E=|\tilde{E}|$. Figures (2), (3) and (4) illustrate the dependence of the field gain on the reduced undulator length $\hat{z}$ and the detuning parameter $\hat{\mathrm{C}}$ for $\hat{\Lambda}_{p}^{2} \rightarrow 0,1,4$ respectively.

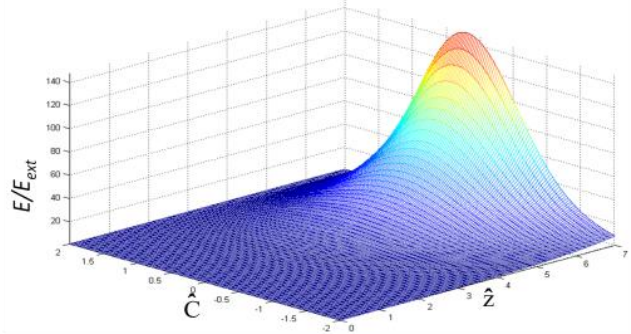

Fig.2. Field gain $E / E_{\text {ext }}$ versus the detuning parameter $\hat{\mathrm{C}}$ and the reduced length of undulator $\hat{z}$. Here $\hat{\Lambda}_{p}^{2} \rightarrow 0$ and $\hat{\Lambda}_{T}^{2} \rightarrow 0$.

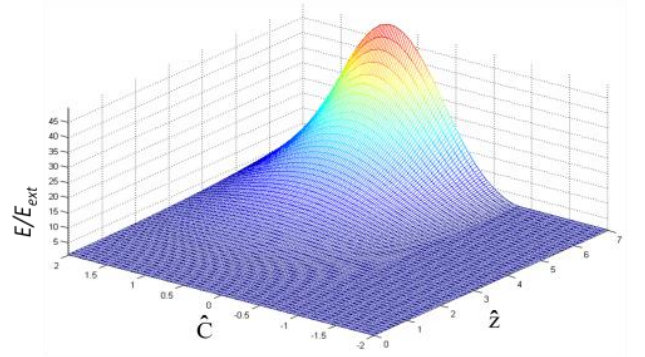

Fig.3. Field gain $E / E_{\text {ext }}$ versus the detuning parameter $\hat{\mathrm{C}}$ and the reduced length of undulator $\hat{z}$. Here $\hat{\Lambda}_{p}^{2} \rightarrow 1$ and $\hat{\Lambda}_{T}^{2} \rightarrow 0$. 


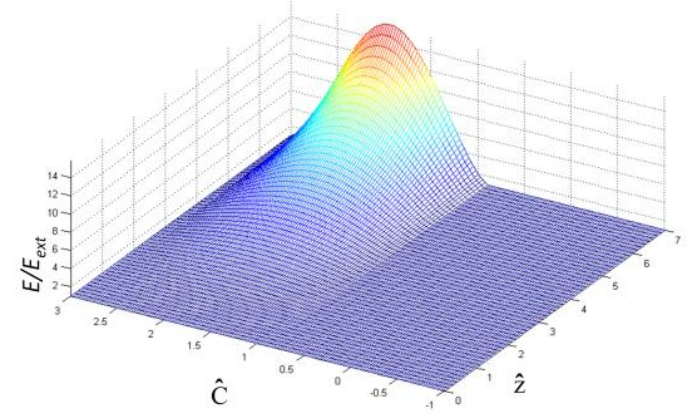

Fig.4. Field gain $E / E_{\text {ext }}$ versus the detuning parameter $\hat{\mathbf{C}}$ and the reduced length of undulator $\hat{z}$. Here $\hat{\Lambda}_{p}^{2} \rightarrow 4$ and $\hat{\Lambda}_{T}^{2} \rightarrow 0$.

The demonstration of eigen values solution for Eq.(10) can be presented in Figure (5) and the projection of this figure in front-view plane describe the dependence of maximal reduced field growth rate on the value of space charge parameter which can be shown in Figure (6).

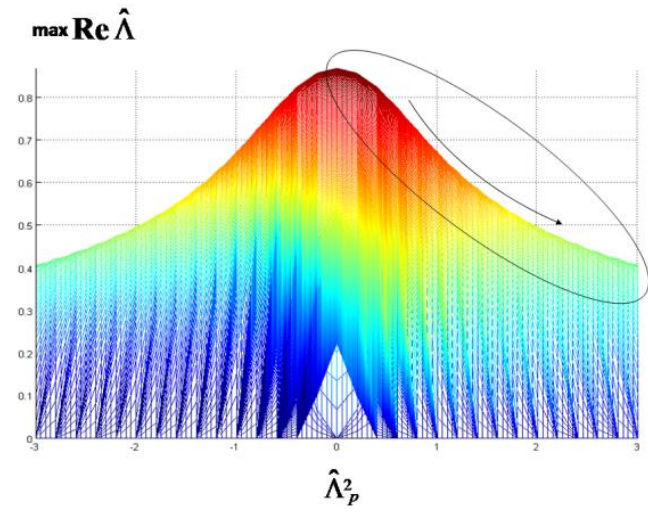

Fig.5. Reduced field growth rate $\operatorname{Re} \hat{\Lambda}$ versus the detuning parameter $\hat{\mathrm{C}}$ and space charge parameter $\hat{\Lambda}_{p}$. Here $\hat{\Lambda}_{T}^{2} \rightarrow 0$.

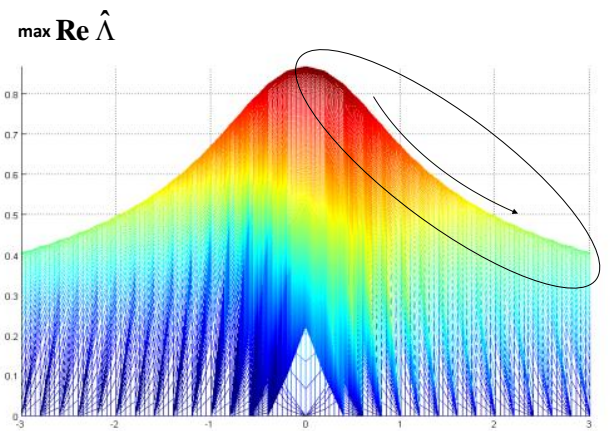

$\hat{\Lambda}_{p}^{2}$

Fig. 6. maximal reduced field growth rate $\max (\operatorname{Re} \hat{\Lambda})$, versus the space charge parameter $\hat{\Lambda}_{p}$. Here $\hat{\Lambda}_{T}^{2} \rightarrow 0$.

\section{CONCLUSION}

The rigorous results obtained in reduced form furnish universal plots for calculation the output characteristics of the FEL amplifier in the linear mode of operation. These solutions serve as a reliable basis for the development of numerical methods.

The analysis of nonlinear processes refers to problems solvable only numerically by a computer. On the other hand, testing of the numerical simulation codes would be difficult without the use of rigorous results of FEL amplifier linear theory as a primary standard.

\section{NOMENCLATURE}

c Speed of the light.

$\mathrm{C}$ Detuning.

$\hat{\mathrm{C}}$ Reduced detuning.

E Amplitude of the electric field.

$\mathrm{E}_{\mathrm{o}} \quad$ Parameter of saturation field amplitude.

E Electric field.

$E_{\text {ext }}$ Amplitude of master signal.

$\tilde{E} \quad$ Complex amplitude of the electric field.

$\tilde{E}_{\text {ext }}$ Complex amplitude of the electric field of the input wave.

$\tilde{E}_{x, y, z}$ Complex amplitude of the electric field components in Cartesian coordinates.

$\hat{E}_{e x t} \quad$ Normalized initial field amplitude.

$\varepsilon \quad$ Energy.

$\varepsilon_{\mathrm{o}} \quad$ Nominal energy of an electron.

$\varepsilon_{\mathbf{o}} \quad$ Permittivity of free space $=8.854 \times 10^{-12} \mathrm{~F} / \mathrm{m}$

-e $\quad$ Charge of the electron $=1.6021892 \times 10^{-19}$ C

$F(P)$ Distribution function of the canonical momentum.

$f \quad$ Distribution function in phase space.

$f_{o} \quad$ Unperturbed distribution function.

$K \quad$ Undulator parameter.

$k_{\omega} \quad$ Wavenumber of the undulator.

$m_{e} \quad$ Electron rest mass $=9.11 \times 10^{-27} \mathrm{~kg}$.

$n_{o} \quad$ Unperturbated particle density of an electron beam. 
$p_{z} \quad$ Longitudinal component of the generalized momentum of the particle.

$P \quad$ Canonical momentum.

$\hat{P} \quad$ Reduced canonical momentum.

$x, y, z$ Cartesian coordinates.

$\hat{x}, \hat{y}, \hat{z} \quad$ Reduced Cartesian coordinates.

$\beta_{x, y, z}$ Relativistic velocity in $x, y$ and $z$ direction respectively.

$\Gamma \quad$ Gain factor in FEL amplifier theory.

$\gamma \quad$ Relativistic factor.

$\mu_{\mathrm{o}} \quad$ Permeability of free space $=4 \pi \times 10^{-7} \mathrm{H} / \mathrm{m}$.

$\eta \quad$ Efficiency.

$\hat{\eta} \quad$ Reduced Efficiency.

$\hat{\Lambda}$ reduced eigenvalue.

$\Lambda_{p} \quad$ Longitudinal plasma wavenumber.

$\hat{\Lambda}_{p}^{2} \quad$ Space charge parameter.

$\lambda \quad$ Radiation wavelength.

$\lambda \omega \quad$ Undulator period.

$\lambda_{j} \quad j^{\text {th }}$ root of an eigenvalue equation.

$\omega \quad$ Frequency of the amplified electromagnetic wave.

$\omega_{\mathrm{o}} \quad$ Resonance frequency.

\section{REFERENCES}

[1] Kim K. J., Three-Dimensional Analysis of Coherent Amplification and Self-Amplified Spontaneous Emission in Free Electron Lasers, Phys. Rev. Let., Vol. 57, pp. 1871-1874, 1986.

[2] Reiche S., Numerical Studies for a Single Pass High Gain Free-Electron Laser, Tech. Rep. DESY thesis-00-012, Deutsches Electronen Synchrotron, (2000).

[3] Zangrando M., Abrami A., Bacescu D., Cudin I., Fava C., Frassetto F., Galimberti A., Godnig R., Giuressi D., Poletto L., Rumiz L., Sergo R., Svetina C., and Cocco D., The photon analysis, delivery, and reduction system at the FERMI@Elettra free electron laser user facility, Rev. Sci. Instrum. 80, 113110 (2009).

[4] Saldin E., Schneidmiller E., Yurkov M., The Physics of Free Electron Lasers, Springer, New York, 1 ed., 1999.

[5] Hesham F. and Minoru Y., Analysis of saturation phenomena in Cerenkov free-electron lasers with a planar waveguide, Physics of Plasmas 18, 093106 (2011).

[6] William H., Saul T., William V., and Brian F., Numerical Recipes: The Art of Scientific Computing in $\mathrm{C}++^{+}, 3$ rd edition by Cambridge University press (2007).

[7] Wang G., Litvinenko, V., Webb S., The Physics of FEL in an Infinite Electron Beam, proceedings of FEL, Malmo, Sweden pp. 60-63, (2010).

[8] Ayvazyan V., et al., A new Powerful Source for Coherent VUV Radiation: Demonstration of Exponential Growth and Saturation at the TTF Free Electron Laser, Eur. Phy. J., D20, pp.149156, 2002.

[9] Markus H., Surface Roughness Wake Fields and Longitudinal Phase Space in a Linear Electron Accelerator, Tech. Rep. DESY thesis-00-020, Deutsches Electronen Synchrotron, (2002).

[10] Group of MathWork, Symbolic Math Toolbox User's Guide, 7th edition by The MathWorks, Inc., (2008). 


\section{إستخلاص الحل العام للمعادلة التكاملية_التفاضلية في حزمة إكترون كاوسين}

$$
\begin{aligned}
& \text { حيدر الجبوري1، محمد الفايز²، رائد خميس3 } \\
& 1 \text { كلية علوم الحاسب و الرياضيات، جامعة تكريت، العراق }
\end{aligned}
$$

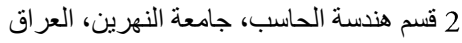

$$
\begin{aligned}
& 3 \text { قسم العلوم التطبيقية ، جامعة التكنولوجيا، العر اق }
\end{aligned}
$$

الملخص:

أصبح الأساس النظري لمنظومات ليزر الإلكترون الحر من المبادئ الراسخة ويمكنها ان تقدم وصفا و افيا لطيف و اسع من الاختبار ات. حيث ان البحوث جارية لأنظمة تعمل من واحد مليمتر وتصل الى نحو عشرة نانو متر من الطول الموجة بالاستتاد على النظريات ذاتها.

في هذا العمل، النتائج النهائية لتوزيع طاقة لورنزية في مضخم ليزر الإلكترون الحر سنوجد بحل المعادلة التكاملية_التفاضلية حيث استعملت خصائص وميزات للغة البرمجة (MATLAB Scientific Language) في هذا البحث. علاوة على ذلك تم وصف العلاقة بين عدة عو امل لاستخلاص وتمثيل العلاقات المركبة بين عدد من عوامل ليزر الإلكترون الحر. 